\title{
The aqueous Raman optical activity spectra of 4(R)-hydroxyproline: theory and experiment
}

\author{
Magdalena Pecul, a,b* Christine Deillon, ${ }^{c}$ Andreas J. Thorvaldsen ${ }^{b}$ \\ and Kenneth Ruud
}

\begin{abstract}
Vibrational Raman optical activity (ROA) spectra have been measured for aqueous solutions of $4(R)$-hydroxyproline at three different $\mathrm{pH}$ values and are compared with theoretical results calculated for several conformations of anionic, cationic and zwitterionic $4(R)$-hydroxyproline using density functional theory (DFT) and the polarizable continuum model (PCM). The experimental ROA bands have been ascribed to the normal modes by comparison of the experimental and calculated vibrational frequencies and ROA intensities. Overall, using PCM for geometry optimization and force field calculations gives simulated Raman and ROA spectra that agree with the main features of the experimental spectra, whereas using PCM also in the calculations of optical tensors seems more problematic.
\end{abstract}

Keywords: Raman optical activity; amino acids; $a b$ initio calculations; polarizable continuum model; pH effects

\section{Introduction}

Vibrational Raman optical activity (ROA) spectroscopy is based on the phenomenon of inelastic scattering of circularly polarized light by chiral molecules. It is measured as the difference in intensity of the right and left circularly polarized scattered Raman light. This effect was predicted and its existence demonstrated by Barron et al. ${ }^{[1,2]}$ in the early 1970s. Until recently, the technique was to some extent a scientific curiosity. However, it has been gaining importance in the last few years, especially in the field of biochemistry, partly because of developments in the experimental technique, such as the cancellation of offsets by subtracting the spectra measured for the actual chiral molecule and that of its virtual, optically generated antipode. ${ }^{[3]}$ ROA spectra are very sensitive to molecular conformation and - since the majority of biologically active molecules, and in particular biopolymers, are chiral-ROA spectroscopy is potentially a powerful tool in biophysics, biochemistry and structural biology.

One of the most important applications of ROA is the investigation of protein folding and of structure and dynamics of nucleic acids. Application of ROA in molecular biophysics enables us to characterize transition states of proteins with respect to both basic motifs of their secondary structure and spatial packing. ${ }^{[4-11]}$ ROA spectra are being used to characterize secondary and tertiary structures of protein $s^{[4,11]}$ and there have been attempts to utilize the technique also for nucleic acids. ${ }^{[12,13]}$ However, the relation between the ROA spectrum and the molecular conformation is complex, and it is difficult to derive simple empirical correlations between them. In such cases, ab initio studies can be very helpful.

Another field which calls for cooperation between experimental ROA spectroscopists and computational chemists is the assignment of absolute configurations by means of ROA. ${ }^{[14-16]}$ ROA spectra coupled with theoretical calculations also allow us to obtain additional information on the vibrational spectra, since bands which coalesce in conventional Raman spectra may have different signs in their right and left circular polarization intensities, and therefore be separable in a ROA spectrum.

The first complete $a b$ initio simulation of an ROA spectrum was presented by Polavarapu ${ }^{[17]}$ in 1990 using the static approximation of Amos. ${ }^{[18]}$ The first correlated calculations using multiconfigurational self-consistent field (MCSCF) wave functions and London atomic orbitals were presented 4 years later, ${ }^{[19]}$ where also frequency-dependent optical tensors were employed. Nowadays, other computational techniques are available, including time-dependent density functional theory (DFT) ${ }^{[20,21]}$ Much progress has recently been made in making $a b$ initio calculations of ROA spectra a standard computational tool, $^{[20-27]}$ as reviewed elsewhere. ${ }^{[28-30]}$

The most important factor preventing large-scale $a b$ initio calculations of ROA spectra has been the need for numerical differentiation of optical tensors with respect to nuclear displacements in order to evaluate ROA intensities in the double-harmonic approximation. However, two complementary approaches that allow for analytic calculations of the geometrical derivative of the relevant polarizabilities have recently been presented, first by Champagne and coworkers, ${ }^{[31-33]}$ and very recently also by Thorvaldsen et al. ${ }^{[34,35]}$

ROA intensities, like all chiroptical properties, ${ }^{[36]}$ appear to depend significantly on the molecular environment, ${ }^{[24]}$ in particular

\footnotetext{
* Correspondence to: Magdalena Pecul, Faculty of Chemistry, University of Warsaw, Pasteura 1, 02-093 Warszawa, Poland.E-mail:mpecul@chem.uw.edu.pl

a Faculty of Chemistry, University of Warsaw, Pasteura 1, 02-093 Warszawa, Poland

b Centrefor Theoretical and Computational Chemistry, Department of Chemistry, University of Tromsø, N-9037 Tromsø, Norway

c Department of Physical Chemistry, University of Fribourg, Pérolles, $\mathrm{CH}-1700$ Fribourg, Switzerland
} 
on the solvent. This poses a challenge for theoretical simulations. One technique used to deal with the problem of solvent effects is the polarizable continuum model (PCM), in which a solvent is treated as a uniform, polarizable dielectric continuum. In the present paper, we test the applicability of PCM to simulate aqueous environments. Another point we address by means of the combination of experimental and theoretical methods, is the influence of $\mathrm{pH}$ on the ROA spectra of amino acids.

Amino acids, the building blocks of proteins, are a frequent subject of experimental ROA spectroscopy. The experimental ROA spectra of proline has been recorded in $\mathrm{H}_{2} \mathrm{O}$ and $\mathrm{D}_{2} \mathrm{O}$ solutions by Kapitán et al., ${ }^{[37]}$ and a complete vibrational assignment was suggested on the basis of theoretical calculations. In this article, we present for the first time a combined experimental and theoretical study of the ROA spectra of 4(R)-hydroxyproline. Experimental ROA spectra have been collected for aqueous solutions at three different $\mathrm{pH}$ values. $A b$ initio results have been obtained for a number of conformers of the anionic, cationic and zwitterionic $4(R)$-hydroxyproline. The main purpose of this work is to test the applicability of DFT/Hartree-Fock calculations combined with the PCM model for the solvent in predicting the ROA spectrum of a flexible, fairly complicated and hydrogen-bond-forming molecule.

The remaining part of the paper is organized as follows. First, the theory underlying the calculation of ROA is briefly outlined together with the computational details, followed by the experimental details. The results of the calculations and measurements are reported in the next section: the optimized structures of the molecules under study are described, and the experimental and calculated ROA spectra are discussed. Finally, the results are summarized and some concluding remarks are given.

\section{Methodology}

\section{Theory and computational details}

The measured ROA signal is the difference in intensity of the backward $\left(\pi=180^{\circ}\right)$ scattered signal between right $\left(I_{R}(\pi)\right)$ and left $\left(I_{\llcorner}(\pi)\right)$ circularly polarized light. The difference can be calculated as $^{[1,38]}$

$$
I_{\mathrm{R}}(\pi)-I_{\mathrm{L}}(\pi)=24 \beta\left(G^{\prime}\right)^{2}+8 \beta(A)^{2},
$$

where

$$
\begin{aligned}
\beta\left(G^{\prime}\right)^{2} & =\sum_{i k} \frac{3 \alpha_{k i}^{v} G_{k i}^{v}-\alpha_{k k}^{v} G_{i i}^{\prime v}}{2} \\
\beta(A)^{2} & =\sum_{i j k l} \frac{1}{2} \omega_{\mathrm{rad}} \alpha_{k i}^{v} \epsilon_{k j l} A_{j l i}^{v}
\end{aligned}
$$

$\omega_{\text {rad }}$ is here the radiation's angular frequency and $\epsilon_{k j l}$ is the unit third-rank antisymmetric Levi-Civita tensor. In the presented data, $I_{R}(\pi)-I_{L}(\pi)$ has been multiplied by the Boltzmann factor $\exp (-\hbar \omega / k T)$ (where $\omega$ is the frequency associated with the vibrational transition and $\hbar$ and $k$ are the Planck and Boltzmann constants, respectively) calculated for the temperature $T=298 \mathrm{~K}$. Within the double harmonic approximation, $G_{i j}^{\prime v}$ and $A_{i j k}^{v}$ are derivatives of the imaginary part of the electric dipole-magnetic dipole polarizability $\mathbf{G}^{\prime}$, and the real part of the electricdipole-electric quadrupole polarizability $\mathbf{A}^{[38]}$ with respect to the normal coordinate of the vibration under study.

In the case of the gas-phase calculations, we have used the recently developed atomic orbital-based scheme for analytic calculations of the ROA circular intensity differences. ${ }^{[35,29]}$ The approach is based on a general framework for the calculation of higher-order molecular properties for basis sets which may be both time and perturbation dependent. ${ }^{[34]}$ The approach uses as a starting point the quasi-energy derivative with respect to a nuclear displacement (the quasi-energy gradient), which can be expressed in terms of the density matrix in the atomic-orbital basis as

$$
Q^{g}=\frac{d Q}{d g}=\left\{\frac{\partial \tilde{E}(\mathbf{D})}{\partial g}-\operatorname{Tr}^{g} \mathbf{W}\right\}_{t},
$$

where we have introduced the short-hand notation $A^{b}=\frac{d A}{d b}$ for derivatives, and $\{\ldots\}_{t}$ indicates that we have performed a time averaging over a full period of the applied electromagnetic field (or equivalently: over all time). In the equation, we have also introduced the density matrix $\mathbf{D}$ in the atomic orbital basis, the generalized (time-dependent) self-consistent field (SCF) energy of the system, $\tilde{E}(\mathbf{D})$, its transposed partial derivative, the generalized (time-dependent) Fock matrix in the AO basis $\tilde{\mathbf{F}}$, and the energy-frequency-weighted density matrix $\mathbf{W}$, all quantities defined as

$$
\begin{aligned}
\tilde{E}(\mathbf{D}) & =h_{\text {nuc }}+v_{\text {nuc }}+\operatorname{Tr}\left(\mathbf{h}-\frac{i}{2} \mathbf{T}+\mathbf{V}\right) \mathbf{D}+\operatorname{Tr} \frac{1}{2} \mathbf{G ( D ) D ,} \\
\tilde{\mathbf{F}} & =\frac{\partial \tilde{E}(\mathbf{D})}{\partial \mathbf{D}^{T}}=\mathbf{h}+\mathbf{V}-\frac{i}{2} \mathbf{T}+\mathbf{G}(\mathbf{D}), \\
\mathbf{W} & =\mathbf{D} \tilde{\mathbf{F}} \mathbf{D}+\frac{i}{2} \dot{\mathbf{D}} \mathbf{S} \mathbf{D}-\frac{i}{2} \mathbf{D S} \dot{\mathbf{D}}, \quad \dot{\mathbf{D}}=\frac{d}{d t} \mathbf{D},
\end{aligned}
$$

respectively. $\mathbf{S}$ is here the conventional overlap matrix, $\mathbf{S}^{g}$ the geometry-differentiated overlap matrix, and $\mathbf{T}$ an antisymmetric time-differentiated overlap matrix

$$
\begin{aligned}
& S_{\mu \nu}^{g}=\frac{\partial S_{\mu \nu}}{\partial g}=\frac{\partial}{\partial g}\left\langle\chi_{\mu} \mid \chi_{\nu}\right\rangle_{,} \\
& T_{\mu \nu}=\left\langle\chi_{\mu} \mid \dot{\chi}_{\nu}\right\rangle-\left\langle\dot{\chi}_{\mu} \mid \chi_{\nu}\right\rangle, \quad \dot{\chi}_{\mu}=\frac{d}{d t} \chi_{\mu} .
\end{aligned}
$$

$h_{\text {nuc }}$ is the nuclear repulsion energy, $\mathbf{h}$ is the integral matrix of the conventional one-electron operator (in atomic units)

$$
h_{\mu \nu}=\left\langle\chi_{\mu}\left|-\frac{1}{2} \nabla^{2}-\sum_{K} \frac{Z_{K}}{\left|\mathrm{R}_{K}-\mathrm{r}\right|}\right| \chi_{\nu}\right\rangle
$$

and $\mathbf{G}(\mathbf{D})$ represents the two-electron interaction, which in the atomic orbital basis can be written as

$$
G_{\mu \nu}(\mathbf{D})=\sum_{\rho \sigma} D_{\sigma \rho}\left(g_{\mu \nu \rho \sigma}-g_{\mu \sigma \rho \nu}\right)
$$

with the two-electron integrals being defined as

$$
g_{\mu v \rho \sigma}=\iint \chi_{\mu}^{*}\left(\mathrm{x}_{1}\right) \chi_{\nu}\left(\mathrm{x}_{1}\right) \frac{1}{r_{12}} \chi_{\rho}^{*}\left(\mathrm{x}_{2}\right) \chi_{\sigma}\left(\mathrm{x}_{2}\right) d \mathrm{x}_{1} d \mathrm{x}_{2}
$$

where $x_{i}$ comprises a spin and a spatial coordinate. In Eqn (5), $v_{\text {nuc }}$ is the potential energy between the nuclei and the external fields, while $\mathbf{V}$ describes the interaction between the electrons and the external fields. In the electric quadrupole approximation, the 
monochromatic field-molecule interaction operator $\hat{V}(t)$ entering the Hamiltonian is given by

$$
\begin{aligned}
\hat{V}(t)= & -F(t) \hat{\mu}-Q(t) \hat{\Theta}-B(t) \hat{m} \\
= & -\left(f \exp \left(-i \omega_{\mathrm{rad}} t\right)+f^{*} \exp \left(i \omega_{\mathrm{rad}} t\right)\right) \hat{\mu} \\
& -\left(q \exp \left(-i \omega_{\mathrm{rad}} t\right)+q^{*} \exp \left(i \omega_{\mathrm{rad}} t\right)\right) \hat{\Theta} \\
& -\left(-i b \exp \left(-i \omega_{\mathrm{rad}} t\right)+i b^{*} \exp \left(i \omega_{\mathrm{rad}} t\right)\right) \hat{m},
\end{aligned}
$$

in which the monochromatic electromagnetic wave is represented as an oscillating, inhomogeneous electric field and an oscillating homogeneous magnetic field, determined by corresponding complex-valued frequency component vectors $f, q$ and $b$

$$
\begin{aligned}
& f=\left\{f_{x}, f_{y}, f_{z}\right\}, \\
& q=\left\{q_{x x}, q_{x y}, q_{y y}, q_{x z}, q_{y z}, q_{z z}\right\}, \\
& b=\left\{b_{x}, b_{y}, b_{z}\right\},
\end{aligned}
$$

which we treat as perturbations (perturbation strengths), and which multiply the electric dipole-, electric quadrupole- and magnetic dipole moment operators, respectively. Since $\hat{m}$ is an imaginary operator, a factor $-i$ has been added so that $b$ multiplies the imaginary part of $\hat{m}$, which is a real anti-Hermitian operator. This avoids having imaginary perturbed (differentiated) integrals $\left(\mathbf{V}^{b}\right.$, etc.).

The various polarizability gradients needed for the calculation of the ROA circular intensity differences can now be obtained by straightforward differentiation of the quasi-energy gradient in Eqn 4, as described elsewhere. ${ }^{[35]}$ Here we limit ourselves to presenting the final expressions for the different polarizability gradients

$$
\begin{aligned}
Q^{g f^{*} f} & =\operatorname{Tr}\left\{\mathbf{V}^{g f} \mathbf{D}^{f^{*}}+\mathbf{V}^{g f^{*}} \mathbf{D}^{f}+\mathbf{h}^{g} \mathbf{D}^{f^{*} f}\right. \\
& \left.+\mathbf{G}^{g}\left(\mathbf{D}^{f^{*}}\right) \mathbf{D}^{f}+\mathbf{G}^{g}(\mathbf{D}) \mathbf{D}^{f^{*} f}-\mathbf{S}^{g} \mathbf{W}^{f^{*} f}\right\}_{t}, \\
Q^{f^{*} q} & =\operatorname{Tr}\left\{\mathbf{V}^{g q} \mathbf{D}^{f^{*}}+\mathbf{V}^{g f^{*}} \mathbf{D}^{q}+\mathbf{h}^{g} \mathbf{D}^{f^{*} q}\right. \\
& \left.+\mathbf{G}^{g}\left(\mathbf{D}^{f^{*}}\right) \mathbf{D}^{q}+\mathbf{G}^{g}(\mathbf{D}) \mathbf{D}^{f^{*} q}-\mathbf{S}^{g} \mathbf{W}^{f^{*} q}\right\}_{t}, \\
Q^{f^{*} b} & =\operatorname{Tr}\left\{\mathbf{V}^{g b} \mathbf{D}^{f^{*}}+\mathbf{V}^{g f^{*}} \mathbf{D}^{b}+\mathbf{h}^{g} \mathbf{D}^{f^{*} b}\right. \\
& \left.+\mathbf{G}^{g}\left(\mathbf{D}^{f^{*}}\right) \mathbf{D}^{b}+\mathbf{G}^{g}(\mathbf{D}) \mathbf{D}^{f^{*} b}-\mathbf{S}^{g} \mathbf{W}^{f^{*} b}\right\}_{t} .
\end{aligned}
$$

These expressions contain the first- and second-order perturbed density matrices with respect to the electric field $f\left(\right.$ or $\left.f^{*}\right)$, a magnetic field $b$ and an electric field gradient $q$, respectively. These perturbed density matrices are determined using the linearly scaling response solver of Coriani et al. ${ }^{[39]}$

It is worth noticing that in contrast to other analytic implementations of $\mathrm{ROA}^{[31-33]}$, the equations above utilize the so-called $n+1$ rule instead of the $2 n+1$ rule. In this way, we avoid having to solve equations for a perturbed density matrix for each displacement of the nuclei (of which there are three times the number of the nuclei), limiting the number of perturbed density matrices that need to be determined to 45 , independent of the size of the molecule. These 45 densities are 12 first-order perturbed density matrices ( 3 for the dipole moment operator, 3 for the magnetic dipole moment and 6 for the quadrupole operator using the symmetry of the operator), and 33 determined by means of second-order equations ( 6 second-order in the electric fields, 9 mixed electric-magnetic dipole second-order and 18 mixed electric dipole-electric quadrupole perturbed density matrices).

For the solvent calculations we have used the extension of integral-equation formalism polarizable continuum model IEF$\mathrm{PCM}^{[40-42]}$ for the calculation of ROA circular intensity differences as described by Pecul et al. ${ }^{[24]}$ In this approach, the different electronic polarizabilities are calculated analytically, but the geometrical derivatives of the polarizabilities are obtained by finite displacements of the nuclear positions. Local field effects have not been included, as these can be expected to be of minor importance for properties involving mixed hyperpolarizabilities. ${ }^{[24]}$ Even though the implementation in Ref. [24] allows London orbitals to be utilized, we will only report the numbers obtained without London orbitals in order to facilitate the discussion of the solvent effects, since our analytical approach used for the gas-phase calculations has not yet been extended to allow for the use of London orbitals, even though the formalism has been described in detail in Ref. [29]. We have verified that the difference between London and non-London results is small.

The structures of anionic, cationic and zwitterionic forms of hydroxyproline have been optimized at the DFT(B3LYP)/aug-ccpVDZ level using the Gaussian03 ${ }^{[43]}$ program. Gaussian03 was also used to obtain the vibrational Hessian. The PCM in its integral-equation formalism (IEF-PCM) ${ }^{[40,44-46]}$ with standard van der Waals radii for functional groups (as default in Gaussian03 ${ }^{[43]}$ ) and the $\alpha$ factor set to 1.2 has been used to simulate the aqueous environment. In the case of the anionic and cationic structures, also gas-phase-like optimizations (of the isolated molecules) have been carried out. The optical tensors, for the isolated molecules and in the PCM-simulated aqueous environment, have been calculated using a local version of DALTON. ${ }^{[47]}$ The ROA intensities have been calculated at the Hartree-Fock level of theory using the aug-ccpVDZ basis set.

The vibrational modes have been analyzed in terms of potential energy distribution (PED) using the VEDA program ${ }^{[48]}$ and visualized by means of PyVib2. ${ }^{[49,50]}$ The presented spectra have been simulated by representing each ROA band as a Lorentzshaped curve with a half-width of $5 \mathrm{~cm}^{-1}$.

\section{Experimental details}

Trans-4-hydroxyproline-L-proline was purchased from Fluka. The solutions were filtered with Ultrafree-MC Amicon filters from Millipore $(0.22 \mu \mathrm{m}$ filter unit). The $\mathrm{pH}$ of the almost saturated solutions was adjusted with a micro-electrode. Before the measurements, the cuvette was centrifuged at $1000 \mathrm{~g}$ with an Eppendorf centrifuge. Scattered circular polarization (SCP) ROA backscattering spectra were taken at room temperature, with the exciting wavelength of $532 \mathrm{~nm}$ and a depolarized incident beam. ${ }^{[3]}$ The resolution was $7 \mathrm{~cm}^{-1}$ and the sample size was $35 \mu \mathrm{l}$. Seven-point third-order Savitzky-Golay smoothing was applied.

\section{Results and Discussion}

\section{Molecular structures}

The starting structures for the geometry optimizations have been selected partly on the basis of the gas-phase conformations of neutral $4(R)$-hydroxyproline ${ }^{[51]}$ and cationic and anionic proline, ${ }^{[52]}$ and partly on the basis of the proline structures obtained in Ref. [52]. The optimized minimum structures (as obtained by means of PCM) are shown in Figs 1,2 and 3, while the energy is tabulated in Tables 1, 2, and 3, respectively. In all cases, the conformations are listed according to increasing energy. For anionic and cationic forms of hydroxyproline, we decided to perform the calculations also for gas-phase isolated ions, to allow for a comparison with PCM results. No gas-phase data are listed 


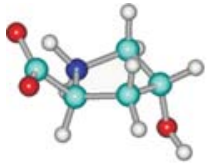

I

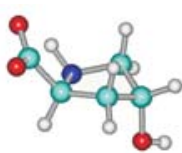

II

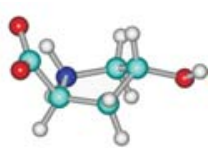

III

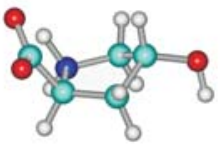

IV

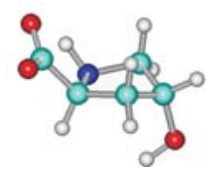

V
Figure 1. The predicted low-energy conformers of zwitterion $4(R)$ hydroxyproline.
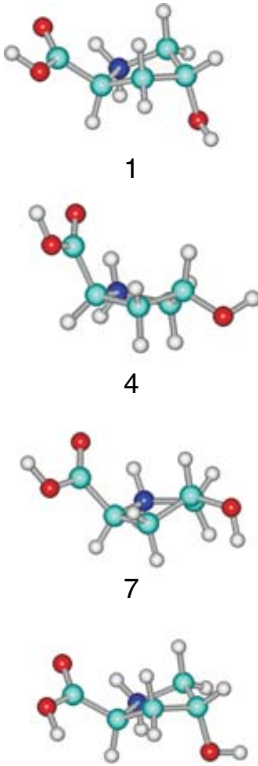

10

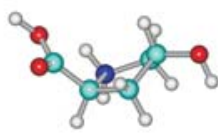

13
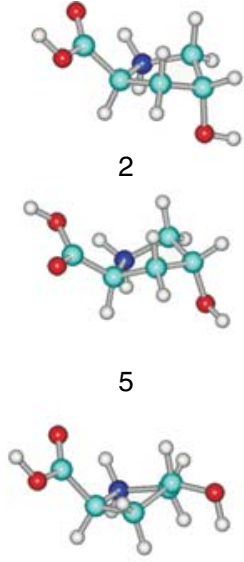

8

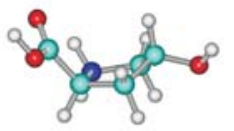

11

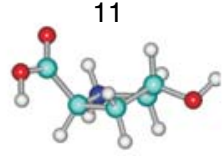

14

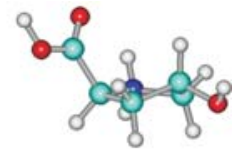

6

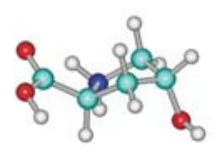

9

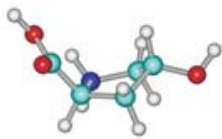

12

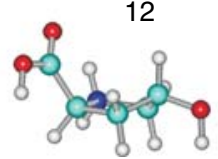

15

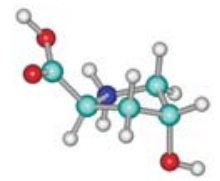

Figure 2. The predicted low-energy conformers of cationic 4(R)hydroxyproline.

for hydroxyproline in the zwitterionic form, since zwitterionic structures are unstable without an aqueous environment.

The two lowest zwitterionic structures (Fig. 1), I and II, both have the endo conformation of the ring and differ only in the arrangement of the hydroxyl group. This is in agreement with the findings of Pecul et al. ${ }^{[52]}$ for neutral and ionic proline and with Kapitan et al. ${ }^{[37]}$ for the zwitterion form, where the forms of proline lowest in energy has endo conformation of the pyrrolidine ring (ring down-puckered with respect to the carboxyl group). The next two structures, significantly higher in energy (Table 1), have the exo form of the ring (ring up-puckered with respect to the carboxyl group). All low-lying structures under consideration have an internal hydrogen bond between the $-\mathrm{NH}_{2}{ }^{+}$and $\mathrm{COO}^{-}$ groups. We have omitted the neutral forms of $4(R)$-hydroxyproline in our subsequent calculations. This omission should not introduce large errors in the following calculations of the ROA spectra, since

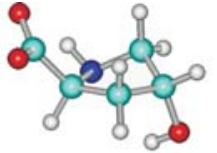

A

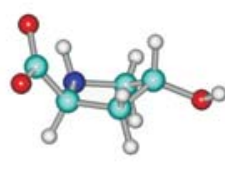

D

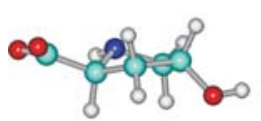

G

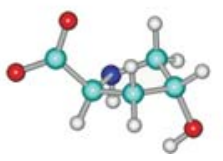

$\mathrm{K}$

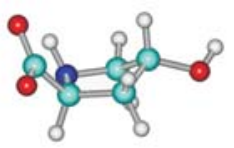

B

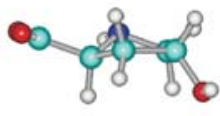

$\mathrm{E}$

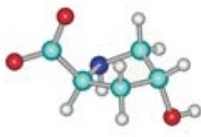

$\mathrm{H}$
C

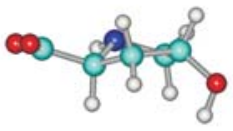

$\mathrm{F}$

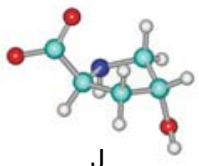

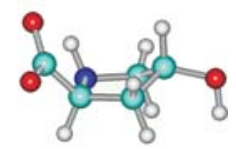

Figure 3. The predicted low-energy conformers of anionic $4(R)$ hydroxyproline.

there is a sizable energy gap between the most stable zwitterionic form and the most stable neutral form in aqueous environment.

The cationic form has two conformations with low energy, again both with the endo form of the ring (Fig. 2). As for the anionic proline, although to a lesser extent, the energy gaps between different conformers are lowered when geometry optimization is carried out using PCM, as shown in Table 2. It is worth noting that there are more minima in the dielectric environment than for the isolated molecules, since for the latter some of them converge to the same structures.

In the case of the anionic form of 4-(R)-hydroxyproline, there is a large energy gap between the four conformations which are lowest in energy and the remaining structures (Table 3). The lowest structure has the endo form of the ring (Fig. 3), while the next three, differing only in the conformation of the hydroxyl group, have the exo form of the ring. When the geometry optimization is carried out using the PCM model, the four lowest lying structures are very close in energy (the energy gap between the first and fourth structure is less than $1 \mathrm{~kJ} / \mathrm{mol}$ ). However, we emphasize that the well-known problems of DFT in the description of diffuse states (including anionic species, see for example Ref. [52]) make the results obtained for anionic hydroxyproline less reliable than for the other forms. This is true not only for the geometries but also for the ROA intensities as discussed below.

\section{ROA spectra}

We will discuss and compare the calculated and experimental ROA spectra of 4(R)-hydroxyproline. Since the comparison with experiment is possible only for wavenumbers lower than $1900 \mathrm{~cm}^{-1}$, the spectra are shown only up to this wavenumber. Arbitrary units are used for the ROA intensities. If not specifically noted, the vibrations described are for the first conformer drawn in Figs 1-3, corresponding to the conformer of lowest energy of the zwitterionic, cationic and anionic forms of 4(R)-hydroxyproline, respectively. The averaged spectra have been calculated using Maxwell-Boltzmann distributions, employing relative energies at 
Table 1. The energy of zwitterion hydroxyproline relative to the lowest conformer, in $\mathrm{kJ} / \mathrm{mol}$. PCM results. $D_{0}$ contains zero-point vibrational correction

\begin{tabular}{|lrl|} 
& $D_{e}$ & $D_{0}$ \\
\hline I & 0.0 & 0.0 \\
II & 0.6 & 0.6 \\
III & 5.1 & 4.5 \\
IV & 7.4 & 7.4 \\
V & 10.4 & 9.2 \\
\hline
\end{tabular}

Table 2. The energy of cationic hydroxyproline relative to the lowest conformer, in $\mathrm{kJ} / \mathrm{mol}$. $D_{0}$ contains zero-point vibrational correction

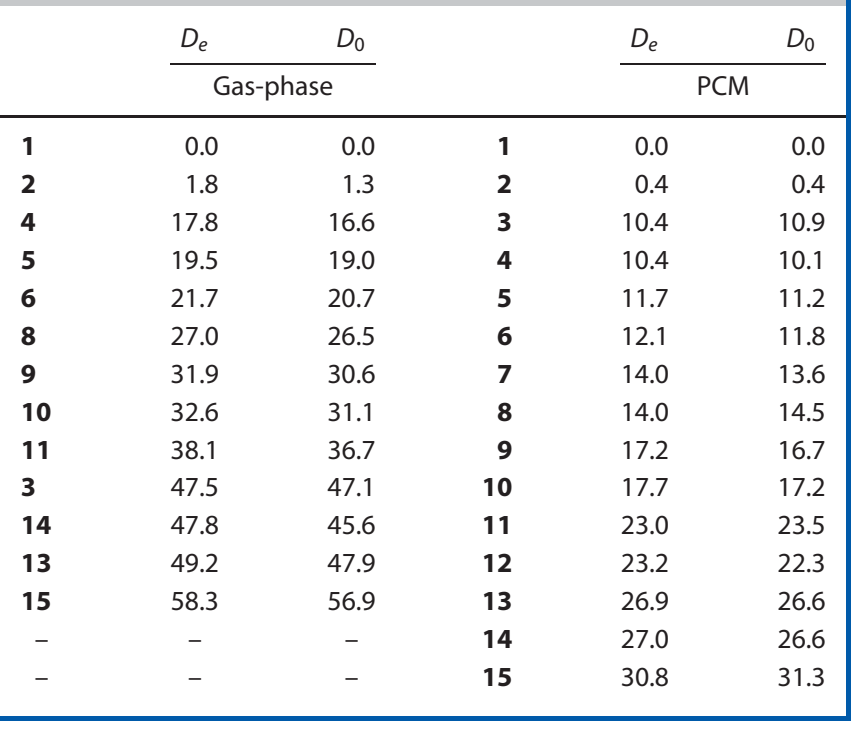

$0 \mathrm{~K}$ (including zero-point vibrational energies). The appearance of the calculated spectra is not changed when free energies evaluated at room temperature are used instead. The experimental peaks are assigned to specific vibrational modes on the basis of comparison with theoretical predictions and PED analysis. The atom numbering used in the following analysis is shown in Fig. 4.

Figure 5 contains the experimental Raman and ROA spectra of 4(R)-hydroxyproline collected for three $\mathrm{pH}$ values. The effect of $\mathrm{pH}$ change on them is not very large: the largest change is the additional band at $1736 \mathrm{~cm}^{-1}$ (visible only in the Raman spectrum) in the spectrum taken at $\mathrm{pH}=1$, corresponding to the stretching of the carboxyl group coupled with the bending vibration of the acidic proton (see below). This spectrum also lacks the band at $1430 \mathrm{~cm}^{-1}$, present in those taken at $\mathrm{pH}=6$ and $\mathrm{pH}=10$, and the sign of the ROA band at $1380 \mathrm{~cm}^{-1}$ is reversed with respect to the other two spectra. It should be mentioned at this point that the effects of $\mathrm{pH}$ may be exaggerated in the theoretical spectra, since we assume there are only cations in the acidic solution and only anions in the basic one, while the actual composition of the solution is more complicated.

\section{Zwitterionic form $\left(\mathrm{NH}_{2}{ }^{+} \mathrm{CHRCOO}^{-}\right)$}

The calculated ROA spectra of the 4(R)-hydroxyproline zwitterion are shown in Fig. 6, the uppermost spectrum obtained by including PCM only for the geometry optimization and force field
Table 3. The energy of anionic hydroxyproline relative to the lowest conformer, in $\mathrm{kJ} / \mathrm{mol}$. $D_{0}$ contains zero-point vibrational correction

\begin{tabular}{ccccccc} 
& \multicolumn{2}{c}{$D_{e}$} & & & $D_{e}$ & $D_{0}$ \\
\cline { 2 - 6 } & \multicolumn{2}{c}{ Gas-phase } & & & \multicolumn{2}{c}{ PCM } \\
\hline A & 0.0 & 0.0 & $\mathbf{A}$ & 0.0 & 0.0 \\
$\mathbf{C}$ & 8.3 & 6.9 & $\mathbf{B}$ & 0.5 & 0.6 \\
$\mathbf{D}$ & 9.5 & 8.2 & $\mathbf{C}$ & 0.6 & 1.0 \\
$\mathbf{B}$ & 12.1 & 10.8 & $\mathbf{D}$ & 0.7 & 0.7 \\
$\mathbf{F}$ & 28.2 & 25.2 & $\mathbf{E}$ & 11.3 & 10.2 \\
$\mathbf{E}$ & 32.1 & 29.0 & $\mathbf{F}$ & 12.0 & 11.0 \\
$\mathbf{G}$ & 35.7 & 32.4 & $\mathbf{G}$ & 12.5 & 11.0 \\
$\mathbf{J}$ & 39.5 & 37.7 & $\mathbf{H}$ & 13.3 & 13.3 \\
$\mathbf{H}$ & 41.2 & 39.9 & $\mathbf{J}$ & 13.5 & 13.4 \\
- & - & - & $\mathbf{K}$ & 16.7 & 16.1 \\
\hline
\end{tabular}

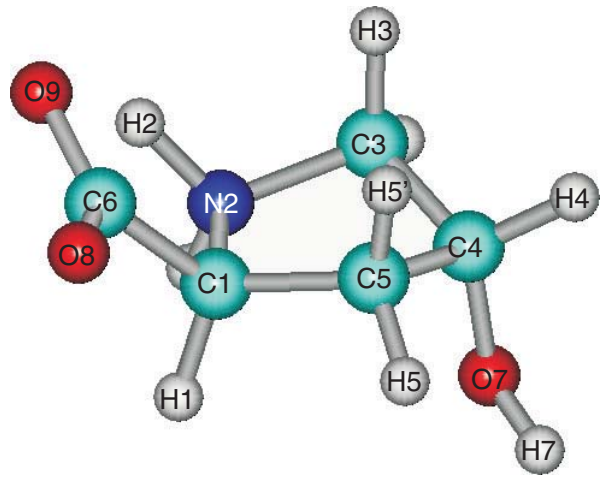

Figure 4. Atom numbering in 4(R)-hydroxyproline.

(PCM/gas) and the one in the middle with the optical tensors also calculated using PCM (PCM/PCM). The experimental spectrum collected at $\mathrm{pH}=6$, where the zwitterionic conformations are dominant, is shown below for comparison. The calculated spectra originate predominantly (88\%) from the two lowest zwitterionic conformations (I and II).

The most striking feature of the theoretically obtained spectra is that treatment of the solvent by means of PCM increases the ROA intensities of the $v(C-H)$ stretching vibrations (with large ROA intensity, not shown in Fig. 6) by one order of magnitude. ROA intensities of the other vibrations are also increased (in terms of their absolute value) when placing the molecule in the dielectric medium, but to a lesser extent. However, the instances where the use of PCM for the optical tensors change the sign of the ROA intensity of a vibration are rare.

Rather surprisingly, the calculated spectrum is closer to experiment when PCM is used only in the geometry and force field calculations (the PCM/gas spectrum), especially in the region below $1400 \mathrm{~cm}^{-1}$, than when PCM is used also for the calculations of optical tensors. Below, we ascribe the most active ROA bands to the specific vibrations on the basis of a PED analysis.

The theoretically predicted $v\left(\mathrm{COO}^{-}\right)$antisymmetric stretching vibration with some $\mathrm{NH}_{2}$ bending has a wavenumber of $1674 \mathrm{~cm}^{-1}$ in both of the leading conformations (to be compared with the experimentally observed wavenumber of $1645 \mathrm{~cm}^{-1}$ ), and a negative ROA activity (strong in conformer I, weak in conformer II). Close to it $\left(1596 \mathrm{~cm}^{-1}\right.$ in the theoretical spectrum, $1582 \mathrm{~cm}^{-1}$ in the experimental spectrum) there is a band corresponding 


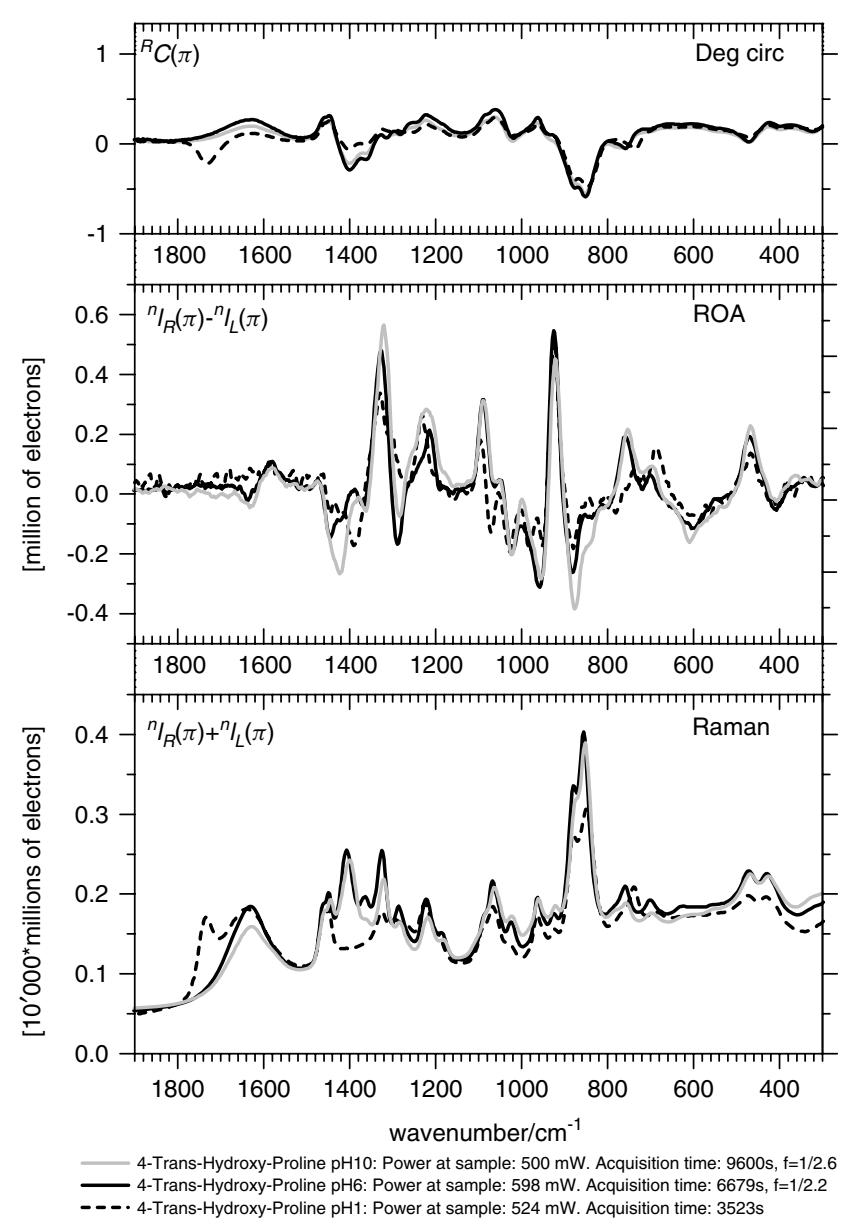

Figure 5. The experimental ROA, Raman and degree of circularity spectra of $4(R)$-hydroxyproline $(100 \mathrm{mg} / \mathrm{ml})$ collected in $\mathrm{H}_{2} \mathrm{O}$ at $\mathrm{pH}=1,6$ and 10 .

to the $\delta\left(\mathrm{NH}_{2}\right)$ bending vibration with a small contribution from the $v\left(\mathrm{COO}^{-}\right)$antisymmetric stretching vibration with a very weak ROA intensity, which disappears almost completely in $\mathrm{D}_{2} \mathrm{O}$ (see Fig. S1 of the Supporting Information). The contribution of the bending vibration is larger here than in the one at $1645 \mathrm{~cm}^{-1}$. The calculated ROA intensity of $v(C-O)$ at $1674 \mathrm{~cm}^{-1}$ is overestimated in comparison to experiment, whereas that of $\delta\left(\mathrm{NH}_{2}\right)$ at $1596 \mathrm{~cm}^{-1}$ is somewhat underestimated. The ROA intensity of $\delta\left(\mathrm{NH}_{2}\right)$ is positive in experiment, whereas the calculations give a positive value only for one conformer and only when PCM is used for both force field and optical tensors. The weak negative experimental band at $1491 \mathrm{~cm}^{-1}$ can be identified as an in-phase $\delta\left(\mathrm{CH}_{2}\right)$ scissoring mode of large amplitude on both C5 and C3. It is theoretically predicted at $1484 \mathrm{~cm}^{-1}$, also with a negative ROA intensity, in the PCM/PCM spectrum. The in-phase scissoring mode is followed by an out-of-phase mode, giving a weak positive peak at $1475 \mathrm{~cm}^{-1}$, to which corresponds a medium-strong negative band predicted in the theoretical spectrum at $1472 \mathrm{~cm}^{-1}$. The region from 1473 to $1360 \mathrm{~cm}^{-1}$ shows strong pH dependence (Fig. 5). The most negative experimental peak, at $1449 \mathrm{~cm}^{-1}$, is present at all three $\mathrm{pH}$ values. Surprisingly, it does not have a corresponding theoretical peak for neither conformer I nor II. The experimental negative peak of medium intensity at $1412 \mathrm{~cm}^{-1}$ (weakly negative at $1404 \mathrm{~cm}^{-1}$ in the theoretical spectrum) corresponds primarily to a stretching of the $\mathrm{C} 4-\mathrm{C} 5$ bond coupled with strong, in-phase deformations of the hydrogens on C4 and 07. This vibration is of
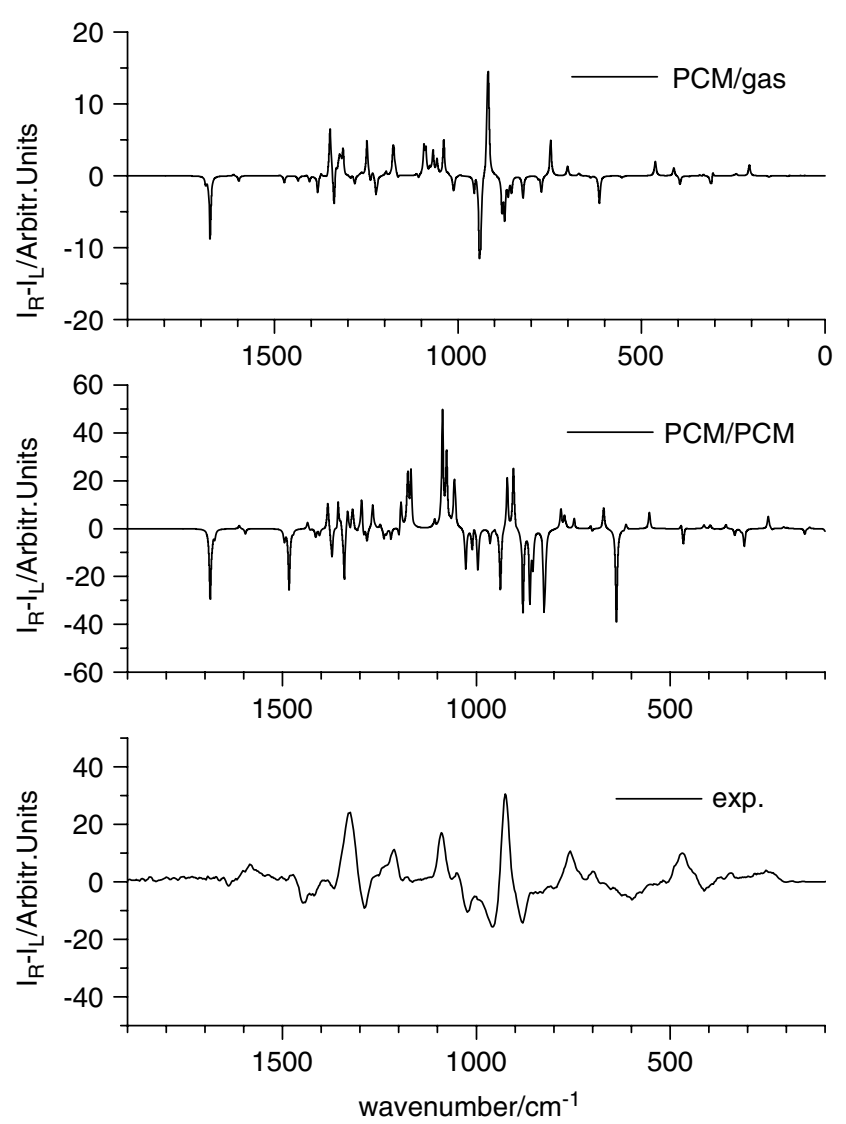

Figure 6. The calculated Boltzman-averaged ROA of the zwitterion 4(R)hydroxyproline (in arbitrary units) and the experimental ROA spectrum of 4(R)-hydroxyproline (less than $100 \mathrm{mg} / \mathrm{ml}$ ) collected at $\mathrm{pH}=6$, acquisition time: 5679 s, power at sample $598 \mathrm{~mW}$.

comparable experimental intensity at $\mathrm{pH}=1$, more negative at $\mathrm{pH}=10$ and also more negative for the ROA spectrum of proline at this wavenumber (see Fig. S1 of Supporting Information).

The band at $1328 \mathrm{~cm}^{-1}$ in the experimental spectrum with a very large positive ROA activity is probably an overlap of the theoretical peaks at 1332,1324 and $1318 \mathrm{~cm}^{-1}$ (as both the PCM/gas and $\mathrm{PCM} / \mathrm{PCM}$ spectra indicate). The vibration at $1332 \mathrm{~cm}^{-1}$ contains contributions from the $\delta\left(\mathrm{NH}_{2}\right)$ bending, a $v\left(\mathrm{COO}^{-}\right)$symmetric stretch, a weak $\mathrm{C}_{3} \mathrm{H}_{2}$ twisting and a $\mathrm{C}_{5} \mathrm{H}_{2}$ wagging, a $\mathrm{C} 6-\mathrm{C} 1$ and $\mathrm{C} 1-\mathrm{C} 5$ antisymmetric stretch and a deformation of the hydrogen on the two asymmetric carbons $C^{*} 1, C^{*} 4$ and on 07 (the $\mathrm{O} 7-\mathrm{H} 7$ deformation is weak). Deformations of the $\mathrm{C}-\mathrm{H}$ bonds on asymmetric carbons very often give strong ROA signals. Such a deformation is also present at $1324 \mathrm{~cm}^{-1}$, but the movements of the two hydrogens are in a plane situated at about $90^{\circ}$ to the plane which contains the deformations of the hydrogens at $1332 \mathrm{~cm}^{-1}$. The direction of these $\mathrm{C}^{*} \mathrm{H}$ deformations is identical at 1332 and $1318 \mathrm{~cm}^{-1}$. The vibration at $1324 \mathrm{~cm}^{-1}$ shows a stronger deformation of $\mathrm{H} 7$ than the vibration at $1332 \mathrm{~cm}^{-1}$, without a $\mathrm{C} 1-\mathrm{C} 6$ but with a $\mathrm{C} 4-\mathrm{C} 5$ stretching component.

The origin of the next experimental negative ROA band at $1289 \mathrm{~cm}^{-1}$ is less certain: it is probably present as two peaks in the PCM/PCM spectrum and as one peak in the PCM/gas spectrum, and it may have (in conformer I) a contribution from the deformation of the hydrogen on the $\mathrm{C}^{*} 1, \mathrm{C}^{*} 4$ and $\mathrm{O} 7$ atoms, some stretching of the $\mathrm{C} 1-\mathrm{C} 6$ bond, some $\mathrm{NH}(\mathrm{H})$ deformation and some wagging of the $\mathrm{C} 5-\mathrm{H} 2$ moieties. The positive peak at $1212 \mathrm{~cm}^{-1}$ (negative 
at $1220 \mathrm{~cm}^{-1}$ in the theoretical spectrum) represents mostly the twisting of $\mathrm{C}_{3} \mathrm{H}_{2}, \mathrm{C}_{5} \mathrm{H}_{2}$ and $\mathrm{NH}_{2}$ moieties with some deformation of the hydrogen on the asymmetric $C^{*} 1$ and $C^{*} 4$ carbon atoms. This peak disappears almost completely in the $\mathrm{D}_{2} \mathrm{O}$ spectrum (see Fig. S1 of Supporting Information).

The 'pure' $\delta(\mathrm{H} 1-\mathrm{C} 1-\mathrm{C} 6)$ bending vibration has positive ROA intensity as predicted by the calculations (wavenumbers of 1173 or $1176 \mathrm{~cm}^{-1}$, depending on the conformer), and it may correspond to the positive experimental ROA peak of very weak intensity at $1191 \mathrm{~cm}^{-1}$. The negative ROA intensity of proline at this wavenumber is stronger (see Supporting Information). The next experimental peak of medium to strong positive intensity is at $1090 \mathrm{~cm}^{-1}$; it probably corresponds to the strongest positive theoretical peak at $1087 \mathrm{~cm}^{-1}$ in the PCM/PCM spectrum: a stretching of the $\mathrm{C} 4-\mathrm{O} 7$ and $\mathrm{C} 1-\mathrm{C} 5$ bond simultaneously with a twisting of the $\mathrm{C}_{3} \mathrm{H}_{2}, \mathrm{C5H}_{2}$ and $\mathrm{NH}_{2}$ groups. The small experimental peaks at $1067 \mathrm{~cm}^{-1}$ (negative) and $1051 \mathrm{~cm}^{-1}$ (positive) represent stretching of the pyrrolidine ring and of the $\mathrm{C} 4-\mathrm{O} 7$ bond.

The negative peak of medium intensity, present for all $\mathrm{pH}$ values at $1023 \mathrm{~cm}^{-1}$, does not have a corresponding theoretical vibration in conformer I and II. The next sizable ROA band in the experimental spectrum is that at $1010 \mathrm{~cm}^{-1}$ (weakly positive). A comparison with the theoretical spectra allows us to identify it as an asymmetric stretching vibration of the $\mathrm{C} 1-\mathrm{N} 2-\mathrm{C} 3$ ring fragment. The strongest negative experimental peak at $958 \mathrm{~cm}^{-1}$ can be related to the theoretical negative one at $955 \mathrm{~cm}^{-1}$, being a partial ring breathing without movement of the $\mathrm{N} 2-\mathrm{C} 1$ bond. The very strong positive ROA band at $925 \mathrm{~cm}^{-1}$ in the experimental spectrum covers two distinct vibrations in the PCM/PCM spectrum, which represents the experimental spectrum better than the $\mathrm{PCM} / \mathrm{gas}$ spectrum in the region between 958 and $880 \mathrm{~cm}^{-1}$. The first band $\left(938 \mathrm{~cm}^{-1}\right)$ is a stretching of the $\mathrm{N} 2-\mathrm{C} 1, \mathrm{C} 1-\mathrm{C} 5, \mathrm{C} 1-\mathrm{C} 6$ bonds with the $\mathrm{C} 3$ atom immobile. The second is composed of two antisymmetric stretchings of the $\mathrm{N} 2-\mathrm{C} 3$ and $\mathrm{C} 5-\mathrm{C} 4$ bonds. The next negative band at a wavenumber of $880 \mathrm{~cm}^{-1}$, in good agreement with the theoretical spectrum, is a $\mathrm{C} 1-\mathrm{C} 5$ bond stretching $\left(853 \mathrm{~cm}^{-1}\right)$ and a C3-C4 bond stretching $\left(879 \mathrm{~cm}^{-1}\right)$, both vibrations coupled with torsional movements of the atoms in the ring.

The experimental positive ROA band at $757 \mathrm{~cm}^{-1}$ could be described as an out-of-plane bending mode of the $\mathrm{COO}^{-}$group predicted at $747 \mathrm{~cm}^{-1}$ in the theoretical spectrum (or an $\mathrm{H} 1 \mathrm{C} 1 \mathrm{C} 6$ rocking mode), according to the results obtained with PCM used only for the geometry optimization and force field calculations, whereas the small positive ROA band at $700 \mathrm{~cm}^{-1}$ seems to come from the bending of $\mathrm{O} 8 \mathrm{C} 1 \mathrm{C} 4$ and of a torsional movement of the $\mathrm{N} 2-\mathrm{C} 3$ and N2-C1 bonds, N2 moving alternatively in and out the ring.

The medium-weak positive peak at $469 \mathrm{~cm}^{-1}$ corresponds to the theoretical $\delta(\mathrm{C} 6 \mathrm{C} 108)$ bending mode at $462 \mathrm{~cm}^{-1}$, with a deformation of 07 from the $\mathrm{C} 4-\mathrm{O} 7$ bond in phase with the bending; this vibration is also present at $\mathrm{pH}=10$ at $466 \mathrm{~cm}^{-1}$ and at $\mathrm{pH}=1$ at $440 \mathrm{~cm}^{-1}$. In this motion, all atoms of the pyrrolidine ring move as a block, carried by the bending motion; the peak is negative in the PCM/PCM spectrum and positive in the $P C M / g a s ~ s p e c t r u m$. The experimental peak at $413 \mathrm{~cm}^{-1}$ (negative) could correspond to the theoretical peak at $412 \mathrm{~cm}^{-1}$, which is principally a rocking of $\mathrm{C}_{3} \mathrm{H}_{2}$ and $\mathrm{C}_{5} \mathrm{H}_{2}$ with deformation of the hydrogen in $\mathrm{O} 7-\mathrm{H} 7$. The strong deformation of the hydrogen on 07, almost parallel to the ring, predicted theoretically at $331 \mathrm{~cm}^{-1}$ (weak negative in the PCM/PCM spectrum and weak-positive in the $\mathrm{PCM}$ /gas spectrum), is present at $347 \mathrm{~cm}^{-1}$ as a weak positive peak in the experimental spectrum. Summarizing, the largest positive
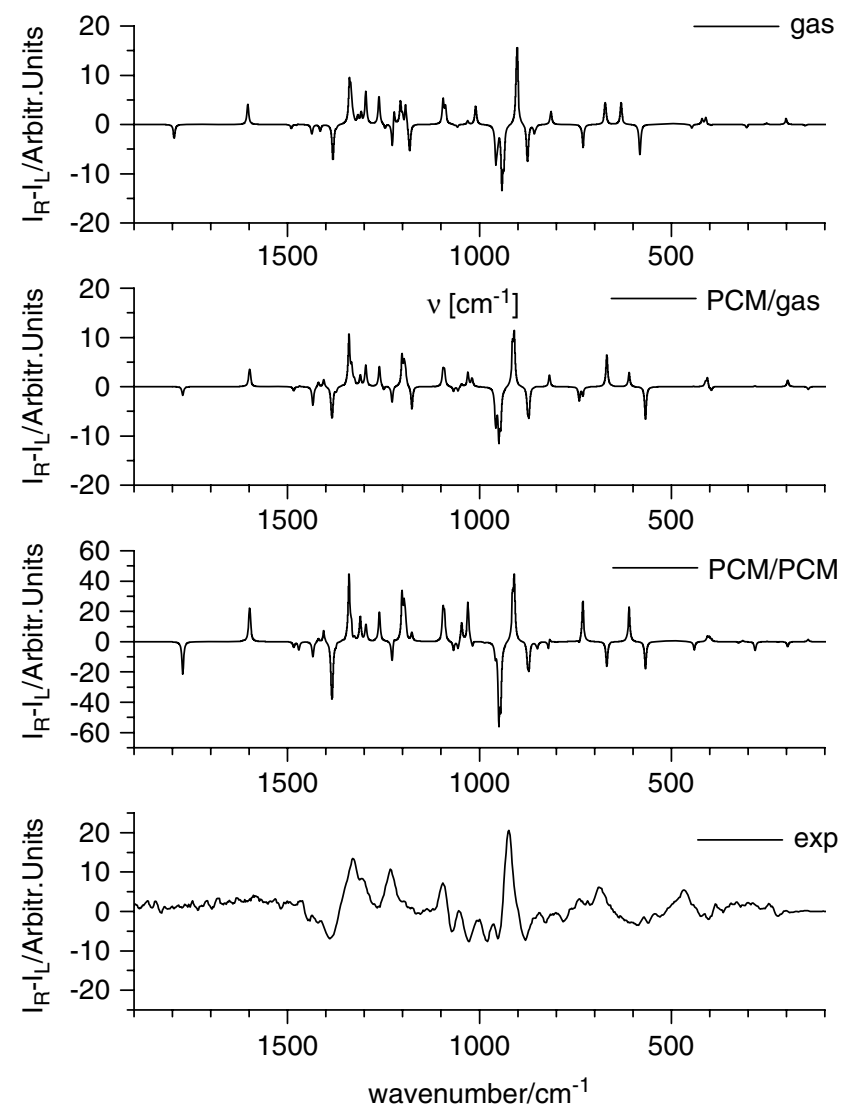

Figure 7. The calculated Boltzman-averaged ROA of the cationic 4(R)hydroxyproline (in arbitrary units) and the experimental ROA spectrum of $4(R)$-hydroxyproline $(100 \mathrm{mg} / \mathrm{ml})$ collected at $\mathrm{pH}=1$, acquisition time: $3523 \mathrm{~s}$, power at sample $524 \mathrm{~mW}$.

and negative ROA intensities are exhibited by the deformation of the hydrogen atom on the asymmetric $C^{*} 1$ and $C^{*} 4$ atoms and the stretching vibrations of the five-membered ring of hydroxyproline.

As far as the conventional Raman spectrum is concerned, the agreement of the calculated and experimental spectrum (Fig. S2 in Supporting Information) is much better than in the case of ROA, especially when PCM is used only for geometry optimization and force field calculations. However, using PCM also for dipole polarizability tensor increases Raman intensities by one order of magnitude, and, similar to ROA, worsens the agreement with experiment.

\section{Cationic form $\left(\mathrm{NH}_{2}{ }^{+} \mathrm{CHRCOOH}\right)$}

The calculated ROA spectra of the cationic form of hydroxyproline and the experimental ROA spectrum collected for $\mathrm{pH}=1$ are shown in Fig. 7. The atom numbering used in this section is as in Fig. 4, and the proton $\mathrm{H} 8$ is defined to be attached to $\mathrm{O} 8$. The calculations have been carried out for an isolated cation (the uppermost graph, denoted 'gas'), employing PCM for geometry optimization and force field only (the second graph, denoted 'PCM/gas'), and employing PCM for geometry optimization, force field and optical tensors (the third graph, denoted ' $P C M / P C M$ '). In the case of the cation, two conformations ( 1 and 2 ) dominate the averaged ROA spectrum. Unlike in the case of the zwitterion, it is difficult to say which of the PCM/gas and PCM/PCM spectra is in better agreement with experiment. 
When the experimental spectrum is analyzed starting from the highest wavenumber, one can observe that the $v(C=0)$ stretching vibration associated with an $\mathrm{O} 8-\mathrm{H} 8$ deformation and some $\mathrm{NH}_{2}$ bending motions, predicted to have small negative ROA intensity in the PCM/gas approach and much larger in the PCM/PCM approach at $1790 \mathrm{~cm}^{-1}$, is not visible at all in the experimental ROA spectrum. However, it is present at $1736 \mathrm{~cm}^{-1}$ in the degree of circularity and Raman spectrum (see Fig. 5). The experimental, weak, positive ROA peak at $1589 \mathrm{~cm}^{-1}$ is a strong $\delta\left(\mathrm{NH}_{2}\right)$ bending mode present at $1600 \mathrm{~cm}^{-1}$ in the PCM/gas and PCM/PCM spectra, identical as at $\mathrm{pH}=6$. The weak negative experimental band centered at $1488 \mathrm{~cm}^{-1}$ can be identified as an in-phase $\delta\left(\mathrm{CH}_{2}\right)$ scissoring mode of hydrogen atoms on both $\mathrm{C} 5$ and C3. It is theoretically predicted at $1483 \mathrm{~cm}^{-1}$, with a weak negative ROA intensity in the PCM/PCM spectrum. The in-phase scissoring mode is followed by an out-of-phase mode of weak positive intensity at $1470 \mathrm{~cm}^{-1}$ in the experimental spectrum, to which corresponds a weak negative band predicted at the same wavenumber.

The theoretical PCM/PCM spectrum shows good agreement with experiment as far as the next discernible ROA bands from 1460 to $1350 \mathrm{~cm}^{-1}$ in the experimental spectrum are concerned, except for the sign of the theoretical peak at $1419 \mathrm{~cm}^{-1}$. The negative peak at $1433 \mathrm{~cm}^{-1}$ originates from a combination of an antisymmetric stretch of $\mathrm{O} 8-\mathrm{C} 6-\mathrm{C}^{*} 1$ with an $\mathrm{H} 8-\mathrm{O} 8-\mathrm{C} 1$ bending, deformations of the hydrogens on $C^{*} 1$ and $C^{*} 4$ (small contribution), an $\mathrm{NH}_{2}$ twisting and a $\mathrm{C}_{3} \mathrm{H}_{2}$ wagging (small contribution). The negative peak of medium-weak intensity at $1426 \mathrm{~cm}^{-1}$ in the experimental spectrum probably corresponds to a theoretical peak at $1419 \mathrm{~cm}^{-1}$, which is a combination of $v(\mathrm{C} 3-\mathrm{C} 4)$ stretching, an in-phase deformation of $\mathrm{H} 7$ and $\mathrm{H} 4$, an in-phase wagging of $\mathrm{NH}_{2}$ and $\mathrm{C}_{3} \mathrm{H}_{2}$ and a rocking of $\mathrm{C}_{5} \mathrm{H}_{2}$. The peak at $1382 \mathrm{~cm}^{-1}$, the most negative peak of all of these, does not have any contribution from the $\mathrm{COO}^{-}$symmetric stretch found in the zwitterionic and anionic forms, as the group is protonated. In the cationic form, the peak at this wavenumber corresponds to an $\mathrm{NH}_{2}$ wagging, a $\mathrm{C}_{3} \mathrm{H}_{2}$ twisting and deformation of the hydrogen atoms bound to 08,07 (small contribution), $\mathrm{C}^{*} 1$ and $\mathrm{C}^{*} 4$.

The large positive experimental ROA band which culminates at $1325 \mathrm{~cm}^{-1}$ seems to contain three theoretically calculated peaks at about 1340, 1331 and $1310 \mathrm{~cm}^{-1}$ both in the PCM/PCM and PCM/gas spectra. All of these bands result from a strong deformation of the hydrogens on both chiral centers $C^{*} 1$ and $C^{*} 4$. The theoretical vibration at $1340 \mathrm{~cm}^{-1}$ for conformer 1 corresponds additionally to a twisting of $\mathrm{C}_{3} \mathrm{H}_{2}$, a wagging of $\mathrm{C}_{5} \mathrm{H}_{2}$, a $\mathrm{C} 1-\mathrm{C} 5$ stretching and a deformation of the hydrogens on $\mathrm{O} 8$ and 07 . The vibration at $1310 \mathrm{~cm}^{-1}$ stems from an antisymmetric stretching of $\mathrm{C} 4-\mathrm{C} 5$ and $\mathrm{C} 1-\mathrm{C} 5$, a rocking of $\mathrm{C}_{3} \mathrm{H}_{2}$, a twisting of $\mathrm{C}_{5} \mathrm{H}_{2}$ and $\mathrm{NH}_{2}$ (small contribution) and a weak deformation of the hydrogens on 08 and 07 . The vibration at $1331 \mathrm{~cm}^{-1}$ is an $\mathrm{H} 8-\mathrm{O} 8$ deformation with a $\mathrm{C} 6-\mathrm{O} 8$ stretching, a wagging of $\mathrm{C}_{3} \mathrm{H}_{2}$ and $\mathrm{C}_{5} \mathrm{H}_{2}$ and a weak rocking of $\mathrm{NH}_{2}$. All vibrations from 1340 to $1250 \mathrm{~cm}^{-1}$ are $\mathrm{pH}$-dependent, with particularly strong dependence of those visible in the experimental spectrum from 1310 to $1250 \mathrm{~cm}^{-1}$ (Fig. 5).

The experimental $\mathrm{pH}$-specific shoulder at $1296 \mathrm{~cm}^{-1}$ corresponds to a theoretical positive peak of similar intensity at the same wavenumber. It contains components from a $\mathrm{C}_{5} \mathrm{H}_{2}$ wagging, a C4-C5 stretching, an in-phase displacement of one hydrogen on $\mathrm{NH}_{2}$ and of another on the opposite side of the ring on $\mathrm{C}_{3} \mathrm{H}_{2}$, and deformations of the hydrogen atoms in the $\mathrm{C}^{*} 1-\mathrm{H}, \mathrm{C}^{*} 4-\mathrm{H}$ and $\mathrm{O} 8-\mathrm{H} 8$ bonds. The experimentally determined peak of very low intensity at $1266 \mathrm{~cm}^{-1}$ may correspond to a theoretically predicted positive peak at $1260 \mathrm{~cm}^{-1}$. It originates from a combination of an $\mathrm{NH}_{2}$ twisting, a $\mathrm{C}_{3} \mathrm{H}_{2}$ rocking and a deformation of the hydrogens on $\mathrm{C}^{*} 1$ (in phase with $\mathrm{C} 5 \mathrm{H} 5 \mathrm{H}^{\prime}$ ), $\mathrm{C}^{*} 4, \mathrm{O} 7$ and $\mathrm{O} 8$ (small contribution).

The next positive ROA band (the third most positive) at $1232 \mathrm{~cm}^{-1}$ seems to correspond to the negative theoretical peak at $1227 \mathrm{~cm}^{-1}$. It has contributions from $\mathrm{H} 5-\mathrm{C} 5-\mathrm{C} 1$ and $\mathrm{H} 7-\mathrm{O} 7-\mathrm{C} 4$ bending modes $\mathrm{C}_{3} \mathrm{H}_{2}$ and $\mathrm{C}_{5} \mathrm{H}_{2}$, antisymmetric twisting and a deformation of the hydrogen on $\mathrm{NH}(\mathrm{H})$ and $\mathrm{O} 8-\mathrm{H} 8$. Almost no vibrations are present in the region between 1200 and $1100 \mathrm{~cm}^{-1}$. The theoretical peaks of medium-strong intensity at about $1200 \mathrm{~cm}^{-1}$ are very weak in the experimental spectrum. The positive peak present experimentally at $1096 \mathrm{~cm}^{-1}$ and predicted theoretically at $1087 \mathrm{~cm}^{-1}$ seems to come from the $v(\mathrm{C} 4-\mathrm{O} 7)$ stretching mode and the stretching of the $\mathrm{C} 1-\mathrm{C} 5$ bond. The next experimental negative peak at $1073 \mathrm{~cm}^{-1}$, of weak to medium intensity, could correspond to the weak negative theoretical peak at $1069 \mathrm{~cm}^{-1}$, which is principally a stretching of the $\mathrm{C} 5-\mathrm{C} 1$, $\mathrm{C} 5-\mathrm{C} 4, \mathrm{~N} 2-\mathrm{C} 4$ and $\mathrm{C} 4-\mathrm{O} 7$ bonds with a rocking of $\mathrm{C}_{3} \mathrm{H}_{2}$. The quite large theoretical negative peak at $1028 \mathrm{~cm}^{-1}$ could correspond to the positive experimental peak at $1030 \mathrm{~cm}^{-1}$ with a wrong sign assignment to this peak, which is of medium intensity in the PCM/PCM spectrum and of weak intensity in the PCM/gas spectrum. The peak at $1030 \mathrm{~cm}^{-1}$ is an antisymmetric stretching of the $\mathrm{N} 2-\mathrm{C} 1-\mathrm{C} 5$ group, a $\mathrm{C} 3-\mathrm{C} 4$ stretch and a rocking of the $\mathrm{C}_{5} \mathrm{H}_{2}, \mathrm{C}_{3} \mathrm{H}_{2}$ and $\mathrm{NH}_{2}$ groups, with deformation of the hydrogen on $C^{*} 4$ and $C^{*} 1$. The negative experimental peak at $984 \mathrm{~cm}^{-1}$ does not have a theoretical counterpart in conformers 1 and 2 .

The most significant difference between theory and experiment is the lack of strong negative experimental ROA bands at approximately $960-949 \mathrm{~cm}^{-1}$, but there is a weak negative peak present at $952 \mathrm{~cm}^{-1}$ in the theoretical spectrum, originating from the pyrrolidine ring breathing with $\mathrm{N}$ being motionless. The strongest positive experimental ROA band at $923 \mathrm{~cm}^{-1}$ probably includes the two strong theoretical peaks (positive at $909 \mathrm{~cm}^{-1}$, an alternative stretching of the N2-C3 and C4-C5 bonds and negative at $949 \mathrm{~cm}^{-1}$, and a stretching of the $\mathrm{C} 1-\mathrm{C} 6$, $\mathrm{C} 4-\mathrm{C} 3, \mathrm{C} 4-\mathrm{O} 7$ and $\mathrm{C} 1-\mathrm{N} 2$ bonds). The pyrrolidine ring breathing has a negative signal of medium intensity at $880 \mathrm{~cm}^{-1}$ in the experimental spectrum and at $870 \mathrm{~cm}^{-1}$ in the theoretical one.

The next region, from 860 to $650 \mathrm{~cm}^{-1}$, shows a distinct experimental profile for the cationic form of trans-4-hydroxy$\mathrm{L}$-proline compared to the anionic and zwitterionic forms. For example, the negative peak at $856 \mathrm{~cm}^{-1}$ in the PCM/PCM spectrum may correspond to the negative shoulder at $856 \mathrm{~cm}^{-1}$ in the experimental spectrum, and stems from a ring breathing with C4-O7 stretching, $\mathrm{NH}_{2}, \mathrm{C}_{5} \mathrm{H}_{2}$ and $\mathrm{C}_{3} \mathrm{H}_{2}$ rocking and a slight deformation of $\mathrm{H} 8$. Some $\mathrm{CO}_{2}$ bending with $\mathrm{O} 8-\mathrm{H} 8$ deformation is present at $820 \mathrm{~cm}^{-1}$. The positive, overestimated peak in the PCM/PCM spectrum at $740 \mathrm{~cm}^{-1}$, positive at $743 \mathrm{~cm}^{-1}$ in the experimental spectrum, comes from a $\mathrm{COO}(\mathrm{H})$ scissoring and is also pH-dependent (Fig. 5).

The origin of the broad positive experimental ROA band at $687 \mathrm{~cm}^{-1}$ is uncertain: it may be a torsional vibration around the $\mathrm{C} 1-\mathrm{C} 6$ bond and/or the $\delta(\mathrm{OCO})$ scissoring mode, including an $\mathrm{O} 8-\mathrm{H} 8$ deformation (such is the nature of the positive PCM/PCM and negative $P C M / g a s$ peaks predicted at $668 \mathrm{~cm}^{-1}$ ). The strong theoretical deformation of the hydroxyl group of the acid moiety, positive at $610 \mathrm{~cm}^{-1}$, coupled with the $\mathrm{COO}(\mathrm{H})$ scissoring, could correspond to the weak negative peak with minimum at $596 \mathrm{~cm}^{-1}$ in the experimental spectrum. The overestimated negative peak at $567 \mathrm{~cm}^{-1}$ in the theoretical spectrum, which is principally a 
strong displacement of $\mathrm{O} 7-\mathrm{H} 7$ without $\mathrm{COO}(\mathrm{H})$ bending but with distortions on the $\mathrm{C} 6-\mathrm{C} 1$ bond, could be matched by the small positive peak at $570 \mathrm{~cm}^{-1}$.

We are not able to identify the positive broad band at $470 \mathrm{~cm}^{-1}$ since there is no substantial ROA-positive band at this wavenumber in any of the theoretical spectra. But if we look at the corresponding wavenumber at $\mathrm{pH}=6$ and $\mathrm{pH}=10$ (see Fig. 5), we have in both cases a medium-weak positive peak at $469 \mathrm{~cm}^{-1}$ which corresponds to the theoretical $\delta(\mathrm{C} 1-\mathrm{C} 6-\mathrm{O} 8)$ bending mode with a deformation of $\mathrm{O} 7$ from the $\mathrm{C} 4-\mathrm{O} 7$ bond in phase with the bending. The weak negative experimental peak at $414 \mathrm{~cm}^{-1}$ could correspond to the positive theoretical peak at $395 \mathrm{~cm}^{-1}$, principally a $\mathrm{C}_{5} \mathrm{H}_{2}$ rocking.

Similar to the case of the zwitterion, the conventional Raman spectrum intensities are in better agreement with experiment than the ROA intensities (see Fig. S3 in Supporting Information). Again, it seems better to use PCM for geometry optimization and force field calculations, but not for calculation of optical tensors.

\section{Anionic form ( $\left.\mathrm{NHCHRCOO}^{-}\right)$}

Figure 8 presents the calculated Boltzman-averaged ROA spectrum of the anionic form of 4(R)-hydroxyproline (in arbitrary units). As for the cation, the theoretical results have been obtained for an isolated anion (upper graph, denoted 'gas'), an anion with force field and Boltzman factors obtained using PCM and optical tensors calculated for an isolated anion (PCM/gas), and an anion in a PCM cavity simulating the aqueous environment (PCM/PCM).
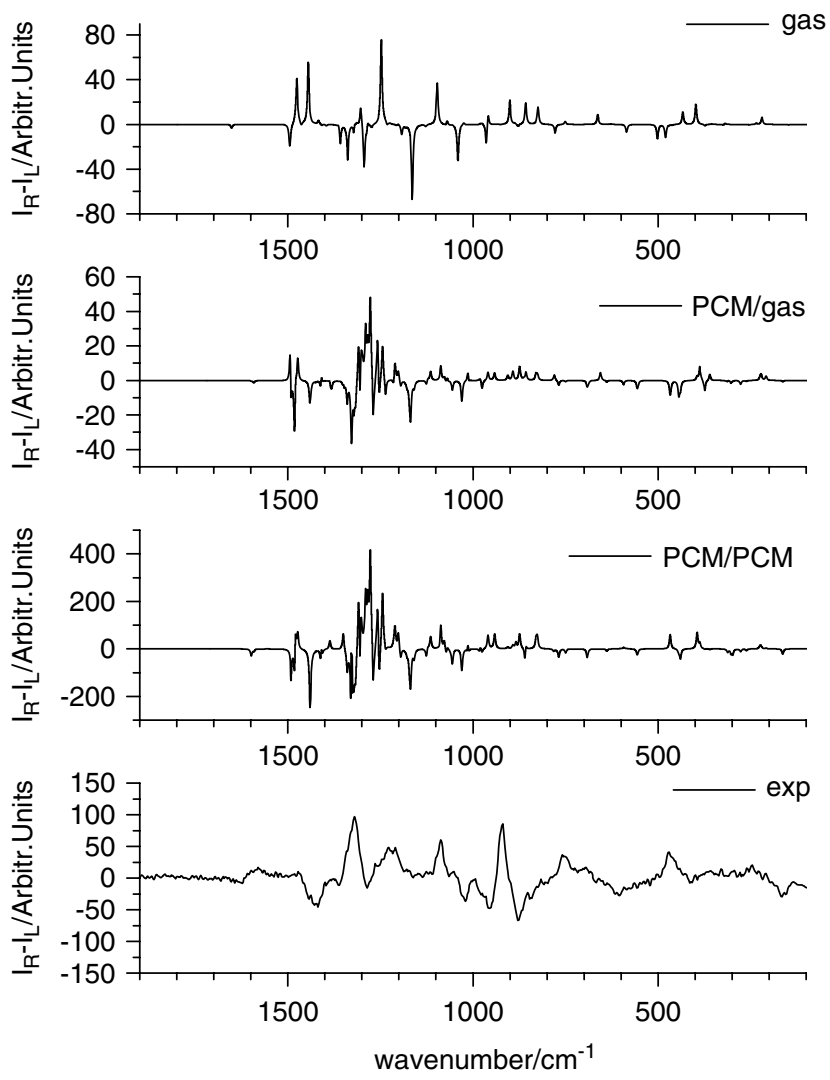

Figure 8. The calculated Boltzman-averaged ROA of the anionic 4(R)hydroxyproline (in arbitrary units) and the experimental ROA spectrum of $4(R)$-hydroxyproline $(100 \mathrm{mg} / \mathrm{ml})$ collected at $\mathrm{pH}=10$, acquisition time: $3993 \mathrm{~s}$, power at sample $500 \mathrm{~mW}$.
In the case of the isolated anion, the spectrum originates in $90 \%$ from conformer $\mathbf{A}$, with an endo conformation of the pyrrolidine ring (the most stable by far, as shown in Table 3 ). In the aqueous environment, there are four low-lying conformers (A, B, C, D) contributing to a comparable degree to the averaged spectrum. B, C and $\mathbf{D}$ are in the exo conformation, and all four conformers have the acid moiety in the same orientation toward the $\mathrm{NH}$ group, allowing for an internal hydrogen bond. These specific positions of $\mathrm{COO}^{-}$and $\mathrm{NH}$ are not found in conformers $\mathbf{E}, \mathbf{F}$ or $\mathbf{G}$. Calculating the optical tensors for the anion placed in a dielectric environment increases the ROA intensities by nearly one order of magnitude (graph 'PCM/PCM' in Fig. 8), and the change is similar for all vibrations. However, the relative intensities in the most interesting region of $900-1500 \mathrm{~cm}^{-1}$ remain approximately the same as in the case when only force field and Boltzman factors have been calculated using PCM (graph 'PCM/gas' in Fig. 8).

The experimental signal with a small positive ROA intensity at $1581 \mathrm{~cm}^{-1}$ originates from an antisymmetric stretching vibration of the $\mathrm{COO}^{-}$group. It has a similar wavenumber in all four lowlying conformers (from 1591 to $1600 \mathrm{~cm}^{-1}$ ), but its ROA intensity is positive in the $\mathbf{B}$ conformer and negative in the remaining three conformers. At the corresponding wavenumbers we have at $\mathrm{pH}=6$ and $\mathrm{pH}=1$ (see Fig. 5) an $\mathrm{NH}_{2}$ bending motion. The antisymmetric stretching of the $\mathrm{COO}^{-}$group of the zwitterionic form contributes primarily to the band at $1645 \mathrm{~cm}^{-1}$.

The two bands just below $1500 \mathrm{~cm}^{-1}$ originate from an inphase (theoretically at $1492 \mathrm{~cm}^{-1}$, experimentally at $1505 \mathrm{~cm}^{-1}$ ) followed by an out-of-phase (theoretically and experimentally at $1473 \mathrm{~cm}^{-1}$ ) scissoring vibration of the $\mathrm{C}_{5} \mathrm{H}_{2}$ and $\mathrm{C}_{3} \mathrm{H}_{2}$ groups. These two vibrations have opposite signs for the ROA intensity in all four low-lying conformers, and those signs differ among the conformers. This, coupled with the substantial ROA activity of these vibrations, creates a complicated signal in the Boltzmannaveraged spectrum. The negative experimental band at $1440 \mathrm{~cm}^{-1}$ is primarily a $\delta(\mathrm{HNC})$ bending vibration, a weak deformation of the hydrogen on $\mathrm{C}^{*} 1, \mathrm{C}^{*} 4$ and $\mathrm{O} 7$ and an in-phase $\mathrm{C}_{3} \mathrm{H}_{2}$ and $\mathrm{C}_{5} \mathrm{H}_{2}$ wagging (small contribution). It is negative in three of the lowlying conformers, and positive only for $\mathbf{A}$. In the weighted averaged spectrum it is negative in both the PCM/PCM and PCM/gas spectra. The next medium-strong negative peak at $1419 \mathrm{~cm}^{-1}$ is present in the experimental spectrum only at $\mathrm{pH}=10$ (Fig. 5). The nearest theoretical positive peak is that at $1406 \mathrm{~cm}^{-1}$, of weak intensity, an out-of-phase deformation of the hydrogens attached to 07 and $\mathrm{C} 4$, with some $\mathrm{NH}$ vibrations and $\mathrm{CH}_{2}$ wagging. The experimental peak we see from 1360 to $1380 \mathrm{~cm}^{-1}$ is positive at $\mathrm{pH}=6$ and $\mathrm{pH}=10$ and negative at $\mathrm{pH}=1$. It corresponds in the theoretical spectrum of the anion to a $\mathrm{COO}^{-}$symmetric stretch coupled to a C1-C4 stretching at $1385 \mathrm{~cm}^{-1}$.

The largest positive band in the experimental spectrum extends from 1364 to $1256 \mathrm{~cm}^{-1}$ with the maximum at $1320 \mathrm{~cm}^{-1}$ and shoulders at 1342 and $1331 \mathrm{~cm}^{-1}$. It corresponds to the multitude of negative and positive theoretical bands present in the PCM/PCM and PCM/gas spectra. The theoretical peak at $1339 \mathrm{~cm}^{-1}$ has a large contribution from a $\mathrm{C}_{3} \mathrm{H}_{2}$ wagging, a stretching of the $\mathrm{C} 1-\mathrm{N}$ and $\mathrm{N} 2-\mathrm{C} 3$ bonds, an $\mathrm{NH}$ deformation and a deformation of the hydrogens on $C^{*} 1$ and $C^{*} 4$. The shoulder at $1331 \mathrm{~cm}^{-1}$ corresponds to the strongest negative theoretical peak and has no theoretical correspondence when looking solely at the spectrum of conformer A. However, there is a vibration with a wavenumber $1328 \mathrm{~cm}^{-1}$ in conformer $\mathbf{C}$, which is a deformation of the hydrogens on $\mathrm{C}^{*} 1$ (strong), $\mathrm{C}^{*} 4$ and $\mathrm{N}, \mathrm{a} \mathrm{C}_{5} \mathrm{H}_{2}$ and $\mathrm{C}_{3} \mathrm{H}_{2}$ twisting and a $\mathrm{C} 4-\mathrm{C} 5$ stretching. The experimental peak 
culminating at $1320 \mathrm{~cm}^{-1}$ could correspond to a large extent to the positive theoretical peak at $1317 \mathrm{~cm}^{-1}$ in conformer $\mathbf{B}$, a deformation of the hydrogens on $\mathrm{C}^{*} 1$ (strong), $\mathrm{C}^{*} 4, \mathrm{~N}$ and O7, a $\mathrm{C5}_{2}$ and $\mathrm{C}_{3} \mathrm{H}_{2}$ wagging and a symmetrical stretching of the $\mathrm{N} 2-\mathrm{C} 1$ and $\mathrm{N} 2-\mathrm{C} 3$ bonds. The weak negative experimental peak at $1286 \mathrm{~cm}^{-1}$ corresponds to the strongest positive peak in the PCM/PCM and PCM/gas spectra at $1280 \mathrm{~cm}^{-1}$, a strong deformation of the hydrogen especially on $\mathrm{C}^{*} 1$ and 07 , but also on $\mathrm{C}^{*} 4$ and $\mathrm{N} 2$ with some additional $\mathrm{C}_{3} \mathrm{H}_{2}$ and $\mathrm{C}_{5} \mathrm{H}_{2}$ wagging for conformer B, C and D.

The next experimental positive ROA peak of medium-strong intensity at $1229 \mathrm{~cm}^{-1}$ is also present at $\mathrm{pH}=1$ but almost absent at $\mathrm{pH}=6$. Looking at the theoretically simulated vibrations, we see in conformer $\mathbf{A}$ a positive peak at $1235 \mathrm{~cm}^{-1}$, a $\mathrm{C} 4-\mathrm{O} 7-\mathrm{H} 7$ bending, an $\mathrm{N}-\mathrm{H}, \mathrm{C}^{*}-4 \mathrm{H}$ and $\mathrm{C}^{*}-1 \mathrm{H}$ deformation, a wagging of $\mathrm{C}_{3} \mathrm{H}_{2}$ and a rocking of $\mathrm{C}_{5} \mathrm{H}_{2}$. The experimental positive peak at $1209 \mathrm{~cm}^{-1}$ is present also at $\mathrm{pH}=6$ but absent at $\mathrm{pH}=1$ and thus should contain a $\mathrm{COO}^{-}$or $\mathrm{NH}$ vibration. However, the nearest theoretical peak, positive at $1195 \mathrm{~cm}^{-1}$, reveals a major $\mathrm{C}_{3} \mathrm{H}_{2}$ twisting with minor $\mathrm{NH}$ deformation. The theoretical band at $1170 \mathrm{~cm}^{-1}$, which comes from another $\delta(\mathrm{HCC})$ bending vibration (of the proton attached to $(4)$, has a strongly negative ROA intensity in conformers $\mathbf{A}$ and $\mathbf{B}$, but is very weak in the measured spectrum.

The next positive experimental peak at $1087 \mathrm{~cm}^{-1}$, with intermediate intensity, corresponds to a positive theoretical peak at the same wavelength in the PCM/PCM and PCM/gas spectra, but not in the gas spectrum, and reflects a torsional mode of the ring ( $\mathrm{C} 1-\mathrm{C} 5$ stretch, conformer $\mathrm{A}$ ), a N2-C3 stretch (conformer $\mathrm{B}$ ) and a deformation of the hydrogen on $C^{*} 4$ and on 07 . This peak differs in intensity from those at $\mathrm{pH} 1$ and 6 , and it does not contain the $\mathrm{NH}_{2}, \mathrm{C}_{3} \mathrm{H}_{2}$ or $\mathrm{C}_{5} \mathrm{H}_{2}$ twisting vibrations.

The subsequent broad peaks, nearly coalescent in the experimental spectrum (positive at 1051, negative at 1020 and negative at $959 \mathrm{~cm}^{-1}$ ), probably correspond to theoretical peaks negative at $1069 \mathrm{~cm}^{-1}$, negative at $1029 \mathrm{~cm}^{-1}$ (the peak of the largest intensity in this series) and positive at $959 \mathrm{~cm}^{-1}$. The vibration at $1069 \mathrm{~cm}^{-1}$, a stretching of the C3-C4 bond, is present in all three theoretical spectra (Fig. 8). The negative vibration at $1026 \mathrm{~cm}^{-1}$ is, at $\mathrm{pH}=10$, an in-phase stretching of the $\mathrm{C} 1-\mathrm{N}$ and $\mathrm{N}-\mathrm{C} 3$ bonds (in conformer $\mathbf{A}$ ) and a partial ring breathing; at $\mathrm{pH}=6$ and 10 the stretching is, according to the results of theoretical calculations, out of phase. The experimental peak at $959 \mathrm{~cm}^{-1}$ is very similar at $\mathrm{pH}=6$ and $\mathrm{pH}=10$. It stems from a ring breathing with an additional stretching of $\mathrm{C} 3-\mathrm{C} 4$ and $\mathrm{C} 4-\mathrm{C} 5$ bonds at $\mathrm{pH}=10$. There is no contribution from nitrogen movement at any of the three $\mathrm{pH}$ values.

The next experimental positive ROA peak culminating at $919 \mathrm{~cm}^{-1}$ with a shoulder at $900 \mathrm{~cm}^{-1}$ is almost as big as the one at $1320 \mathrm{~cm}^{-1}$ and it is identical at all three $\mathrm{pH}$ values. The nearest corresponding peak in the theoretical spectrum is a very weak, positive peak at $905 \mathrm{~cm}^{-1}$, containing a C5-C4 stretching. The experimental negative peak at $879 \mathrm{~cm}^{-1}$ is the strongest negative signal at all wavelengths and any $\mathrm{pH}$. The nearest theoretical positive peak is at $873 \mathrm{~cm}^{-1}$, an antisymmetric stretching of two groups: $\mathrm{C} 1-\mathrm{N}$ and $\mathrm{C} 1-\mathrm{C} 5-\mathrm{C} 4$. The negative peak at $844 \mathrm{~cm}^{-1}$ is negative only at $\mathrm{pH}=10$ : the nearest theoretical positive peak at $830 \mathrm{~cm}^{-1}$ is an OCO bending, coupled with a C6-C1 and $\mathrm{C} 1-\mathrm{N}$ stretching and the torsional movements of the ring. The medium intensity positive peak at $759 \mathrm{~cm}^{-1}$ is absent at $\mathrm{pH}=1$. The corresponding negative theoretical peak at $750 \mathrm{~cm}^{-1}$ in the
PCM/PCM spectrum effectively contains an OCO bending and an antisymmetric motion of $\mathrm{C} 1-\mathrm{C} 5$ and $\mathrm{C} 3-\mathrm{C} 4$.

The last four major experimental peaks are positive at $694 \mathrm{~cm}^{-1}$ (shifted to lower wavenumbers at $\mathrm{pH}=1$ ), negative at $604 \mathrm{~cm}^{-1}$ (stronger at $\mathrm{pH}=10$ ), positive at $473 \mathrm{~cm}^{-1}$ and negative at $414 \mathrm{~cm}^{-1}$; the peak at $694 \mathrm{~cm}^{-1}$ does not have a corresponding theoretical vibration in an anion. In a zwitterion, this vibration is a bending of $\mathrm{O} 8-\mathrm{C} 1-\mathrm{C} 6$ and a torsional movement of the $\mathrm{N}-\mathrm{C} 3$ and $\mathrm{N}-\mathrm{C} 1$ bonds. The next corresponding theoretical peaks are positive at $593 \mathrm{~cm}^{-1}$, (in the $P C M / g a s ~ s p e c t r u m$ ), $466 \mathrm{~cm}^{-1}$ (in the $\mathrm{PCM} / \mathrm{PCM}$ spectrum) and $395 \mathrm{~cm}^{-1}$ (in both spectra). The vibration at $593 \mathrm{~cm}^{-1}$ is to a large extent a C6-C1 stretching with some OCO bending; the vibration at $466 \mathrm{~cm}^{-1}$ is a scissoring motion of $\mathrm{O} 8-\mathrm{C} 6-\mathrm{C} 1$. The vibration at $395 \mathrm{~cm}^{-1}$ is a strong $\mathrm{O} 7-\mathrm{H} 7$ torsional vibration almost parallel to the ring (even stronger at $387 \mathrm{~cm}^{-1}$ and with an antisymmetric rocking of $\mathrm{C}_{3} \mathrm{H}_{2}$ and $\mathrm{C}_{5} \mathrm{H}_{2}$ ).

The anionic form is the only one with an $\mathrm{NH}$ group; we presume that the hydration of this group or the hydrogen bond between the amine and the acid group could have a large influence on the vibration of the molecule, as the anionic theoretical spectrum has the lowest correspondence with the experimental spectrum. Furthermore, as already mentioned, description of anionic species by means of DFT is problematic, and this influences the calculated geometric parameters, energetics and force field. This is also visible in the conventional Raman spectrum, shown in Fig. S4 in Supporting Information: unlike in the case of zwitterion and cationic hydroxyproline, there is little resemblance between the calculated and experimental Raman spectra of anionic 4(R)hydroxyproline.

\section{Summary and Conclusions}

Vibrational ROA spectra have been measured for aqueous solutions of $4(R)$-hydroxyproline for three different $\mathrm{pH}$ values. The experimental spectra collected at $\mathrm{pH}=1,6$ and 10 show significant differences, especially in the region above $1000 \mathrm{~cm}^{-1}$. We have attempted to reproduce these spectra by means of theoretical simulations. The theoretical results have been obtained for several conformations of anionic, cationic and zwitterionic forms of 4(R)-hydroxyproline using DFT and linear response Hartree-Fock calculations. The aqueous environment has been simulated using the IEF-PCM model.

The energetically lowest conformers have an endo form of the ring, as also observed for proline. The ROA spectra calculated for the zwitterionic form stem predominantly (88\%) from the two lowest conformations, and this is also the case for the cation. In the case of the isolated anion, the averaged spectrum originates 90\% from conformer A (by far the most stable, as shown also in Table 3). In the aqueous environment, there are four low-lying conformers (A, B, C, D) contributing to a similar extent to the averaged spectrum.

Whenever possible, assignment of the experimental ROA bands to individual normal modes has been carried out. It has been found that the largest ROA intensities, either negative or positive, are exhibited by stretching vibrations of the five-membered ring of 4-trans-(R)-hydroxyproline. We have tried to analyze whether there is any simple correlation between the ring conformation and the ROA intensity of the ring-stretching vibrations. However, it seems that the total ROA effect is a superposition of the influence of several structural parameters, and no such simple correlation exists. 
The spectra have been modeled in two different ways: with PCM used only for the geometry optimization and force field calculations, and with PCM used also for the calculations of optical tensors. The instances where the use of PCM for optical tensors changes the sign of the ROA intensity of a vibration are rare.

Overall, using PCM only for the geometry optimization and force field calculations seems to give simulated ROA spectra which agree with the main features of the experimental spectra. The agreement is even better in the case of conventional Raman spectra, with the exception of the anionic form. The calculations of optical tensors using PCM seems to be more problematic (particularly for the zwitterionic structures) and seems actually to worsen the agreement with experiment. This may be accidental, since PCM in general is far from being a perfect tool for the simulation of the influence of an aqueous environment on the properties of hydroxyproline, as it does not account for short-range effects such as hydrogen bonds formed by the $\mathrm{COO}^{-}, \mathrm{NH}_{3}{ }^{+}$and $\mathrm{OH}$ groups of $4(R)$-hydroxyproline. It would be therefore of interest to compare the results of PCM calculations with the simulations of ROA spectra by means of molecular dynamics coupled with supermolecular calculations, where hydrogen bonds would be accounted for in an explicit fashion.

\section{Acknowledgments}

The work has been financed by the Ministry of Science and Higher Education (Poland) from funds for scientific research in years 2009-2011 as project No. N N204138637 and by the Norwegian Research Council through a grant of computer time from the Supercomputing Program. KR has also received support from the Research Council of Norway through Grants 179568/V30, $162746 / \mathrm{V} 00$ and $177558 /$ V30. CD received support from the Zürich University (Switzerland) through the Förderung des akademischen Nachwuchses (KFN) and the Stiefel-Zangger-Stiftung (StZ). The measurements were done at the University of Zürich on an instrument designed and constructed by W. Hug, and the authors are grateful to Prof. Hug for allowing us to use this instrument.

\section{Supporting information}

Supporting information may be found in the online version of this article.

\section{References}

[1] L. D. Barron, A. D. Buckingham, Mol. Phys. 1971, 20, 1111.

[2] L. D. Barron, M. P. Bogaard, A. D. Buckingham, J. Am. Chem. Soc. 1973, 95, 603.

[3] W. Hug, Appl. Spectrosc. 2003, 57, 1.

[4] L. D. Barron, L. Hecht, I. H. McColl, E. W. Blanch, Mol. Phys. 2004, 102, 731.

[5] E. W. Blanch, L. Hecht, L. D. Barron, Methods 2003, 29, 196.

[6] F. Zhu, N. W. Isaacs, L. Hecht, L. D. Barron, Structure 2005, 13, 1409.

[7] L. D. Barron, Curr. Opin. Struct. Biol. 2006, 16, 638.

[8] F. Zhu, G. E. Tranter, N. W. Isaacs, L. Hecht, L. D. Barron, J. Mol. Biol. 2006, 363, 19.

[9] L. D. Barron, L. Hecht, E. W. Blanch, A. F. Bell, Prog. Biophys. Mol. Biol. 2000, 73, 1

[10] L. D. Barron, F. Zhu, L. Hecht, G. E. Tranter, N. W. Isaacs, J. Mol. Struct. 2007, 834-836, 7 .

[11] P. Mukhopadhyay, G. Zuber, D. N. Beratan, Biophys.J. 2008, 95, 5574.

[12] A. F. Bell, L. Hecht, L. D. Barron, J. Am. Chem. Soc. 1998, 120, 5820.

[13] L. D. Barron, L. Hecht, A. F. Bell, Appl. Spectrosc. 1996, 50, 619.

[14] P. L. Polavarapu, Chirality 2008, 20, 664.
[15] P. L. Polavarapu, Chem. Rec. 2007, 7, 125.

[16] J. Haesler, I. Schindelholz, E. Riguet, C. Bochet, W. Hug, Nature 2007, 446, 526.

[17] P. L. Polavarapu, J. Phys. Chem. 1990, 94, 8106.

[18] R. D. Amos, Chem. Phys. Lett. 1982, 87, 23.

[19] T. Helgaker, K. Ruud, K. L. Bak, P. Jørgensen, J. Olsen, Faraday Discuss. 1994, 99, 165.

[20] K. Ruud, T. Helgaker, P. Bour, J. Phys. Chem. A 2002, 106, 7448.

[21] P. Bouř, J. Comput. Chem. 2001, 22, 426.

[22] M. Pecul, A. Rizzo, J. Leszczynski, J. Phys. Chem. A 2002, 106, 11008.

[23] M. Pecul, A. Rizzo, Mol. Phys. 2003, 101, 2073.

[24] M. Pecul, E. Lamparska, C. Cappelli, L. Frediani, K. Ruud, J. Phys. Chem. A 2006, 110, 2807.

[25] C. Herrmann, K. Ruud, M. Reiher, Chem. Phys. 2008, 343, 200.

[26] C. Herrmann, K. Ruud, M. Reiher, ChemPhysChem 2006, 7, 2189.

[27] P. Bouř, J.Sopková, L. Bednárová, P. Maloň, T. A. Keiderling, J. Comput. Chem. 1997, 18, 646.

[28] M. Pecul, K. Ruud, Int. J. Quantum Chem. 2005, 104, 816.

[29] A. J.Thorvaldsen, K. Ruud, Chirality 2009, in press. DOI: 10.1002/chir.20777.

[30] M. Pecul, Chirality. in press.

[31] O. Quinet, B. Champagne, J. Chem. Phys. 2001, 115, 6293.

[32] O. Quinet, V. Liégeois, B. Champagne, J. Chem. Theory Comput. 2005, $1,444$.

[33] V. Liegeois, K. Ruud, B. Champagne, J. Chem. Phys. 2007, 127, 204105.

[34] A. J. Thorvaldsen, K. Ruud, K. Kristensen, P. Jørgensen, S. Coriani, J. Chem. Phys. 2008, 129, 214108.

[35] A. J. Thorvaldsen, K. Ruud, M. Fedorovsky, W. Hug, Theor. Chim. Acta. in preparation.

[36] M. Pecul, K. Ruud, Continuum Solvation Models in Chemical Physics, Continuum Polarized Models, Wiley-VCH Chichester 2007.

[37] J. Kapitán, V. Baumruk, V. Kopecký, R. Pohl, P. Bouř, J. Am. Chem. Soc. 2006, 128, 13451.

[38] L.D. Barron, Molecular Light Scattering and Optical Activity, Cambridge University Press: Cambridge, 1982.

[39] S. Coriani, S. Høst, B. Jansik, L. Thøgersen, J. Olsen, P. Jørgensen, S. Reine, F. Pawlowski, T. Helgaker, P. Sałek, J. Chem. Phys. 2007, 126, 154108.

[40] E. Cancés, B. Mennucci, J. Tomasi, J. Chem. Phys. 1997, 107, 3032.

[41] J. Tomasi, M. Persico, Chem. Rev. 1994, 94, 2027.

[42] J. Tomasi, B. Mennucci, R. Cammi, Chem. Phys. 2005, 105, 2999.

[43] M. J. Frisch, G. W. Trucks, H. B. Schlegel, G. E. Scuseria, M. A. Robb, J.R. Cheeseman, J.A. Montgomery Jr, T. Vreven, K. N. Kudin, J.C. Burant, J.M. Millam, S. S. lyengar, J.Tomasi, V. Barone, B. Mennucci, M. Cossi, G.Scalmani, N. Rega, G. A. Petersson, H. Nakatsuji, M. Hada, M. Ehara, K. Toyota, R. Fukuda, J. Hasegawa, M. Ishida, T. Nakajima, Y. Honda, O. Kitao, H. Nakai, M. Klene, X. Li, J. E. Knox, H. P. Hratchian, J. B. Cross, C. Adamo, J. Jaramillo, R. Gomperts, R. E. Stratmann, O. Yazyev, A. J. Austin, R. Cammi, C. Pomelli, J. W. Ochterski, P. Y. Ayala, K. Morokuma, G. A. Voth, P. Salvador, J.J.Dannenberg, V. G. Zakrzewski, S. Dapprich, A.D. Daniels, M.C.Strain, O. Farkas, D. K. Malick, A.D. Rabuck, K. Raghavachari, J. B. Foresman, J. V. Ortiz, Q. Cui, A. G. Baboul, S. Clifford, J.Cioslowski, B. B.Stefanov, G. Liu, A. Liashenko, P.Piskorz, I. Komaromi, R. L. Martin, D. J. Fox, T. Keith, M. A. AlLaham, C. Y. Peng, A. Nanayakkara, M. Challacombe, P. M. W. Gill, B. Johnson, W. Chen, M. W. Wong, C. Gonzalez, J. A. Pople, Gaussian 03, Revision A.1, Gaussian, Inc.: Pittsburgh, PA, 2003.

[44] S. Miertuš, E. Scrocco, J. Tomasi, Chem. Phys. 1981, 55, 117.

[45] R. Cammi, J. Tomasi, J. Comput. Chem. 1995, 16, 1449.

[46] B. Mennucci, E. Cancés, J. Tomasi, J. Phys. Chem. B 1997, 101, 10506.

[47] Dalton, a molecular electronic structure program, Release 2.0, 2005, see http://www.kjemi.uio.no/software/dalton/dalton.html.

[48] M. H. Jamróz, Veda 3, Vibrational energy distribution analysis, title, Veda 3. Vibrational Energy Distribution Analysis.

[49] M. Fedorovsky, PyVib2, a program for analyzing vibrational motion and vibrational spectra, 2007, http://pyvib2.sourceforge.net.

[50] W. Hug, M. Fedorovsky, Theor. Chim. Acta 2008, 119, 113.

[51] A. Lesarri, E. J. Cocinero, J. C. López, J. L. Alonso, J. Am. Chem. Soc. 2004, 127, 2572.

[52] M. Pecul, K. Ruud, A. Rizzo, T. Helgaker, J. Phys. Chem. A 2004, 108, 4269. 